\title{
HNE Interacts Directly with JNK Isoforms in Human Hepatic Stellate Cells
}

\author{
Maurizio Parola, ${ }^{\star}$ Gaia Robino, ${ }^{\star}$ Fabio Marra, ${ }^{\S}$ Massimo Pinzani, ${ }^{\S}$ Giorgio Bellomo, ${ }^{\ddagger}$ Gabriella Leonarduzzi, ${ }^{\star}$ Paola Chiarugi, \\ Simonetta Camandola, ${ }^{\star}$ Giuseppe Poli, ${ }^{\star}$ Georg Waeg, "Paolo Gentilini, ${ }^{\S}$ and Mario Umberto Dianzani* \\ $*$ Dipartimento di Medicina ed Oncologia Sperimentale and ${ }^{\ddagger}$ Dipartimento di Scienze Mediche, University of Torino, Torino, Italy; \\ ${ }^{\S}$ Istituto di Medicina Interna and ${ }^{\|}$Dipartimento di Scienze Biochimiche, Università di Firenze, Firenze, Italy; and "Department of \\ Biochemistry, University of Graz, Graz, Austria
}

\begin{abstract}
4-Hydroxy-2,3-nonenal (HNE) is an aldehydic end product of lipid peroxidation which has been detected in vivo in clinical and experimental conditions of chronic liver damage. HNE has been shown to stimulate procollagen type I gene expression and synthesis in human hepatic stellate cells (hHSC) which are known to play a key role in liver fibrosis. In this study we investigated the molecular mechanisms underlying HNE actions in cultured hHSC. HNE, at doses compatible with those detected in vivo, lead to an early generation of nuclear HNE-protein adducts of 46, 54, and 66 $\mathrm{kD}$, respectively, as revealed by using a monoclonal antibody specific for HNE-histidine adducts. This observation is related to the lack of crucial HNE-metabolizing enzymatic activities in hHSC. Kinetics of appearance of these nuclear adducts suggested translocation of cytosolic proteins. The p46 and p54 isoforms of c-Jun amino-terminal kinase (JNKs) were identified as HNE targets and were activated by this aldehyde. A biphasic increase in AP-1 DNA binding activity, associated with increased mRNA levels of $c$-jun, was also observed in response to $\mathrm{HNE}$. HNE did not affect the Ras/ERK pathway, $c$-fos expression, DNA synthesis, or $\mathrm{NF}-\kappa \mathrm{B}$ binding. This study identifies a novel mechanism linking oxidative stress to nuclear signaling in hHSC. This mechanism is not based on redox sensors and is stimulated by concentrations of HNE compatible with those detected in vivo, and thus may be relevant during chronic liver diseases. (J. Clin. Invest. 1998. 102:1942-1950.) Key words: hepatic stellate cells $\bullet$ HNE adducts • oxidative stress • liver fibrosis • signal transduction
\end{abstract}

\section{Introduction}

Hepatic stellate cells (HSC), ${ }^{1}$ also referred to as Ito cells, lipocytes, or fat-storing cells, are liver-specific pericytes which are known to play a major role in the development of liver fibrosis (1-4). During chronic liver injury HSC transdifferentiate to an

Portions of this work were presented at the 47 th meeting of the American Association for the Study of Liver Disease in Chicago, IL on 8-12 November 1996 and were published in abstract form (1996. Hepatology. 24:1321a).

Address correspondence to Maurizio Parola, Ph.D., Dipartimento di Medicina ed Oncologia Sperimentale, Università di Torino, Corso Raffaello 30, 10125 Torino, Italy. Phone: 39-11-6707752 or 3911-6707754; FAX: 39-11-6707753; E-mail: parola@medfarm.unito.it

Received for publication 5 August 1997 and accepted in revised form 13 October 1998.

J. Clin. Invest.

(C) The American Society for Clinical Investigation, Inc. 0021-9738/98/12/1942/09 \$2.00

Volume 102, Number 11, December 1998, 1942-1950

http://www.jci.org activated myofibroblast-like phenotype which is characterized by marked proliferation and secretion of extracellular matrix components (1-4).

Generally, it is believed that reactive oxygen species (ROS) and other free radicals can play a role in the development of several chronic diseases, including liver fibrosis (5-7). In particular, ROS have been considered relevant since they have been shown to modulate gene expression and cell proliferation through involvement of $c$-jun and $c$-fos and activation of AP-1 and NF-кB (8-11). However, most of these studies have been performed using hydrogen peroxide concentrations (usually $100-300 \mu \mathrm{M}$ ) that cannot be reached in vivo even under conditions of acute oxidative stress (12). In particular, it has been reported recently that administration of an acute oxidative stress to liver parenchyma results in steady-state concentrations of only $0.15 \mu \mathrm{M}$ hydrogen peroxide and no greater than $0.25 \mu \mathrm{M}$ ROS (13).

Hepatic lipid peroxidation, either stimulated by ROS or by prooxidant agents, consists in the oxidative decomposition of $\omega-3$ and $\omega-6$ polyunsaturated fatty acids of membrane phospholipids leading to the formation of a complex mixture of aldehydic end products such as malondialdehyde, 4-hydroxy-2,3nonenal (HNE), and other 4-hydroxy-2,3-alkenals of different chain lengths (14-16). These compounds have been proposed as possible molecular mediators responsible for the close association between lipid peroxidation and enhanced deposition of extracellular matrix reported in vivo and in cell culture systems $(5,7,17,18)$. HNE and 4-hydroxy-alkenals have been detected in vivo in experimental and clinical conditions of chronic liver injury associated with active fibrogenesis, including chronic administration of $\mathrm{CCl}_{4}$ (19), chronic ethanol consumption alone $(20,21)$ or in association with iron (22), extrahepatic cholestasis (23), and primary biliary cirrhosis and chronic hepatitis C (24, 25). HNE and other 4-hydroxy-2,3-alkenals of different chain lengths have been shown to stimulate procollagen type I synthesis in human HSC (hHSC; 26, 27) at concentrations $\left(10^{-6} \mathrm{M}\right)$ which are compatible with a mild to moderate oxidative stress (i.e., lipid peroxidation) occurring in vivo (16). A similar effect has been shown also on rat HSC (6). Moreover, HNE is effective as a profibrogenic stimulus on hHSC at concentrations which are 100-200 times lower than the effective concentrations of malondialdehyde (28), the other major aldehydic end product of lipid peroxidation. Biological effects of HNE strictly depend on its ability to form aldehyde-protein adducts by interacting with sulfhydryl groups or amino groups of lysine and of histidine. In particular, recent studies have emphasized the biological relevance of adducts formed between

1. Abbreviations used in this paper: ALDH, aldehyde dehydrogenase; EMSA, electrophoretic mobility shift assay; hHSC, human HSC; HNE, 4-hydroxy-2,3-nonenal; HSC, hepatic stellate cells; JNKs, c-Jun amino-terminal kinases; LMW-PTP, low molecular weight phosphotyrosine protein phosphatase; MBP, myelin basic protein; NE, nuclear extract; PKC, protein kinase C; ROS, reactive oxygen species. 
HNE and the nitrogen of histidine imidazole ring (16). However, the molecular mechanisms involved in HNE-dependent modulation of gene expression have not yet been defined.

The aim of this study was to elucidate the molecular mechanism underlying HNE action on HSC. We report that HNE forms adducts with c-Jun amino-terminal kinases (JNKs) and that this event leads to JNKs nuclear translocation and activation as well as to $c$-jun and AP-1 induction. These findings identify a novel mechanism linking oxidative stress to nuclear signaling in HSC.

\section{Methods}

Materials. HNE and the monoclonal antibodies (1g4h7) recognizing HNE-histidine adducts specifically have been provided by Prof. Hermann Esterbauer (Department of Biochemistry, University of Graz, Graz, Austria). The monoclonal antibodies $1 \mathrm{~g} 4 \mathrm{~h} 7$ were found to be reliable for immunocytochemistry/immunofluorescence and Western blot applications, but unsuitable for immunoprecipitation. Polyclonal antibodies against JNK isoforms, c-Fos, c-Jun, ATF-2, and recombinant ATF-2 were purchased from Santa Cruz Biotechnology (Santa Cruz, CA). Polyclonal antibodies specifically directed against phosphorylated JNK isoforms were obtained from New England BioLabs (Beverly, MA). All the other reagents were from Sigma Chemical Co. (St. Louis, MO).

Cell isolation and culture. hHSC were isolated from wedge sections of normal human liver tissue unsuitable for transplantation by collagenase-pronase digestion and centrifugation on stractan gradients. Procedures used for cell isolation and characterization have been described extensively elsewhere $(29,30)$. Cells were cultured in Iscove's medium supplemented with $20 \%$ FBS, subcultured when confluent at 1:3 split ratio, and used between passages 4 and 7. Data presented herein were obtained using three separate cell lines.

In all the experiments in which HNE has been used, confluent hHSC were left for $24 \mathrm{~h}$ in serum-free and insulin-free Iscove's medium (SFIF medium) before the addition of the aldehyde in order to obtain quiescent cells and to avoid aspecific binding of HNE to serum proteins. HNE has been used in the 1-10 $\mu \mathrm{M}$ range (i.e., the range detected in vivo), a range which does not exert toxic effects on hHSC, and it has been shown to elicit biologic responses in these cells $(26,27)$.

HNE metabolism in hHSC. The consumption of HNE by hHSC was monitored essentially as described previously (31). In brief, hHSC were incubated in $100-\mathrm{mm}$ petri dishes in the presence of 10 $\mu \mathrm{M}$ HNE for up to $60 \mathrm{~min}$ in $5 \mathrm{ml}$ of SFIF medium. At defined time points an equal volume of ice-cold acetonitrile/acetic acid (96:4, vol/ vol) was added to the dishes in order to inactivate HNE-metabolizing enzymes. Samples were quickly centrifuged at 2,000 $\mathrm{g}$ for $20 \mathrm{~min}$ at $4^{\circ} \mathrm{C}$ to remove protein material and the content of HNE was determined exactly as described previously by means of an HPLC procedure $(31,32)$.

HNE metabolism was further evaluated by determining the following enzymatic activities which are known to be involved in its disposal: aldehyde dehydrogenase (EC 1.2.1.3) was evaluated in cell homogenates; inverse alcohol dehydrogenase (EC 1.1.1.1), glutathione-S-transferase (EC 2.5.1.18), and aldehyde reductase (EC 1.1.1.2) were evaluated in cytosolic fractions. Analytical procedures were exactly as previously described $(31,32)$.

Immunofluorescence and confocal laser microscopy. hHSC were cultured on glass coverslips in SFIF medium for $24 \mathrm{~h}$ and then left untreated or incubated with HNE. At defined time points the cells were fixed with $4 \%$ buffered formalin, $\mathrm{pH} 7.4$, for $10 \mathrm{~min}$ and then permeabilized for 10 min with PBS containing $0.1 \%$ Triton X-100. The cells were then stained for indirect immunofluorescence using the monoclonal antibody $1 \mathrm{~g} 4 \mathrm{~h} 7$, developed against HNE-histidine adducts as previously described (33), as primary antibody at 1:100 (vol/vol) dilution in PBS. A goat anti-mouse FITC-conjugated affinity-purified an- tibody (Sigma, Milano, Italy) was used as secondary antibody at 1:250 dilution ( $\mathrm{vol} / \mathrm{vol})$ in PBS. Cells were viewed with a laser scanner confocal microscope (MRC 600; Bio-Rad, Richmond, CA) equipped with a Nikon diaphot inverted microscope with a $60 / 1.40$ objective. Six focal frames were taken along the $z$ axis at $1-\mu \mathrm{m}$ intervals and then merged to obtain a reconstructed image. To exclude artifacts in the intracellular localization of the antigen, single median focal frames of $1 \mu \mathrm{m}$ were also collected. Fluorescence was transformed in pseudocolors (see reference scale proportional to the intensity of fluorescence). Intracellular localization of fluorescence was also evaluated quantitatively by image analysis at defined time points.

Preparation of cell lysates and nuclear extracts (NEs). Confluent, serum-starved hHSC were treated with the appropriate conditions, quickly placed on ice, and washed with ice-cold PBS. Cell lysates, cytosolic fractions, and NEs were obtained as described (34).

Western blot analysis. Cytosolic fractions and NEs obtained by hHSC were subjected to SDS-PAGE on $10 \%$ acrylamide gels using the mini-PROTEAN II electrophoresis cell (Bio-Rad) according to Laemmli (35). The proteins were transferred electrophoretically to nitrocellulose membranes (Hybond-C extra; Amersham Life Science, Arlington Heights, IL). Unspecific binding was blocked with 5\% (wt/ vol) nonfat dry milk in $50 \mathrm{mM}$ Tris-HCl, pH 7.4, containing $200 \mathrm{mM}$ $\mathrm{NaCl}$ and $0.05 \%$ (vol/vol) Tween 20 (TBS-Tween). The blots were incubated with the different primary antibodies, followed by incubation with peroxidase-conjugated anti-mouse or anti-rabbit immunoglobulins in TBS-Tween containing $1 \%(\mathrm{wt} / \mathrm{vol})$ nonfat dry milk. Immunoblots were developed with the ECL reagents from Amersham according to the manufacturer's instructions.

$J N K$ activity. JNK activity was determined essentially as reported by Lo et al. (36). In brief, proteins were immunoprecipitated with polyclonal anti-JNK antibodies (Santa Cruz Biotechnology). After washing, immunobeads were incubated in reaction buffer $(20 \mathrm{mM}$ Hepes, $\mathrm{pH}$ 7.6, $20 \mathrm{mM} \mathrm{MgCl}$, 2 mM DTT, $25 \mathrm{mM} \beta$-glycerophosphate, and $0.1 \mathrm{mM}$ sodium orthovanadate) containing $20 \mathrm{mM}$ ATP, 5 $\mu \mathrm{Ci}\left[\gamma^{-}{ }^{32} \mathrm{P}\right] \mathrm{ATP}$, and $1 \mu \mathrm{g}$ recombinant ATF-2 (Santa Cruz Biotechnology) for $30 \mathrm{~min}$ at $37^{\circ} \mathrm{C}$. Reaction was stopped by addition of Laemmli buffer and then subjected to $10 \%$ SDS-PAGE. The gel was stained with Coomassie blue, dried, and autoradiographed; bands corresponding to ATF-2 were evaluated by laser densitometry. In some experiments, in order to evaluate the influence of upstream kinases, JNK kinase assay was performed after incubation of immunobeads with recombinant low molecular weight phosphotyrosine protein phosphatase (LMW-PTP, $5 \mu \mathrm{g}$ ) for $3 \mathrm{~min}$ at $37^{\circ} \mathrm{C}$ in a buffer of the following composition: $20 \mathrm{mM}$ Tris- $\mathrm{HCl}, \mathrm{pH} 7.4,150 \mathrm{mM} \mathrm{NaCl}, 5$ $\mathrm{mM}$ EDTA, $1 \mathrm{mM}$ PMSF, and $0.05 \%$ aprotinin. This enzyme, which has been characterized extensively in the past, selectively dephosphorylates phosphotyrosine residues but not phosphoserine or phosphothreonine $(37,38)$. In addition, in vitro studies suggest that LMWPTP is active on different phosphotyrosine substrates with limited specificity in vitro $(37,38)$. This enzyme was chosen in order to avoid treatment with a less specific phosphatase which could bring about dephosphorylation of ATF-2 in the subsequent step.

ERK assay. ERK activity was detected using myelin basic protein (MBP) as substrate. In brief, proteins were immunoprecipitated with polyclonal anti-ERK antibodies (Santa Cruz Biotechnology). After washing, immunobeads were incubated in reaction buffer $(20$ $\mathrm{mM}$ Hepes, $\mathrm{pH}$ 7.4, $1 \mathrm{mM}$ sodium orthovanadate, $1 \mathrm{mM} \mathrm{MgCl}, 1 \mathrm{mM}$ DTT, containing $1 \mathrm{mM}$ ATP, $1 \mu \mathrm{Ci}\left[\gamma^{-32} \mathrm{P}\right] \mathrm{ATP}$, and $0.4 \mathrm{mg} \mathrm{MBP}$ ) for $30 \mathrm{~min}$ at $30^{\circ} \mathrm{C}$. Reaction was stopped by addition of Laemmli buffer and then subjected to $15 \%$ SDS-PAGE. The gel was stained with Coomassie blue, dried, and autoradiographed; bands corresponding to $\mathrm{MBP}$ were evaluated by laser densitometry.

Electrophoretic mobility shift assay (EMSA). Equal amounts of proteins of NEs $(2-5 \mu \mathrm{g})$ were added to a reaction mixture containing $20 \mu \mathrm{g}$ of BSA, $2 \mu \mathrm{g}$ of poly (dI-dC) (Boehringer Mannheim, Mannheim, Germany), $2 \mu$ l of buffer A (20 mM Hepes, $\mathrm{pH} 7.9,20 \%$ glycerol, $100 \mathrm{mM} \mathrm{KCl}, 0.5 \mathrm{mM}$ EDTA, $0.25 \% \mathrm{NP}-40,2 \mathrm{mM}$ DTT, and $0.1 \%$ PMSF), $4 \mu \mathrm{l}$ of buffer B (20\% Ficoll-400, $100 \mathrm{mM}$ Hepes, 300 
$\mathrm{mM} \mathrm{KCl}, 10 \mathrm{mM}$ DTT, and $0.1 \%$ PMSF), and 20,000 cpm of a ${ }^{32} \mathrm{P}-$ labeled oligonucleotide in a final volume of $20 \mu \mathrm{l}$. The reaction mixture contained in addition $5 \mathrm{mM} \mathrm{MgCl} 2$. AP-1, and NF- $\kappa$ B oligonucleotides (Promega, Madison, WI) were labeled using $\left[{ }^{32} \mathrm{P}\right] \mathrm{ATP}(3,000$ $\mathrm{Ci} / \mathrm{mmol}$; Amersham Life Science, Milan, Italy) and $\mathrm{T}_{4}$ polynucleotide kinase (Boehringer Mannheim). After $20 \mathrm{~min}$ at room temperature, the reaction products were separated on a $4 \%$ nondenaturing polyacrylamide gel. Radioactivity of dried gels was detected by exposure to Kodak XAR-54 films and bands were analyzed by laser densitometry.

To identify the composition of AP-1 complex, NEs from HNEtreated cells at 15 and 150 min were either left untreated or preincu- bated with antibodies specifically reacting with c-Jun, c-Fos, and ATF-2, and then used in EMSA as described above.

RNA extraction and Northern blot. For the extraction of cellular RNA, after exposure to HNE, hHSC were washed twice in PBS, pH 7.4 , then scraped with a rubber policeman in a $4 \mathrm{M}$ guanidine isothiocyanate solution, vortexed, and immediately stored at $-80^{\circ} \mathrm{C}$. Total RNA was isolated as described previously (26) following a standard procedure (39) and evaluated spectrophotometrically by the absorbance at $260 \mathrm{~nm}$. Northern blot analysis of mRNA for $c$-jun and $c$-fos was performed according to standard procedure (40) by using cDNA probes (41) which were kindly provided by Dr. Ariane Mallat (INSERM Unité 99, Créteil, France). To exclude significant sample load-
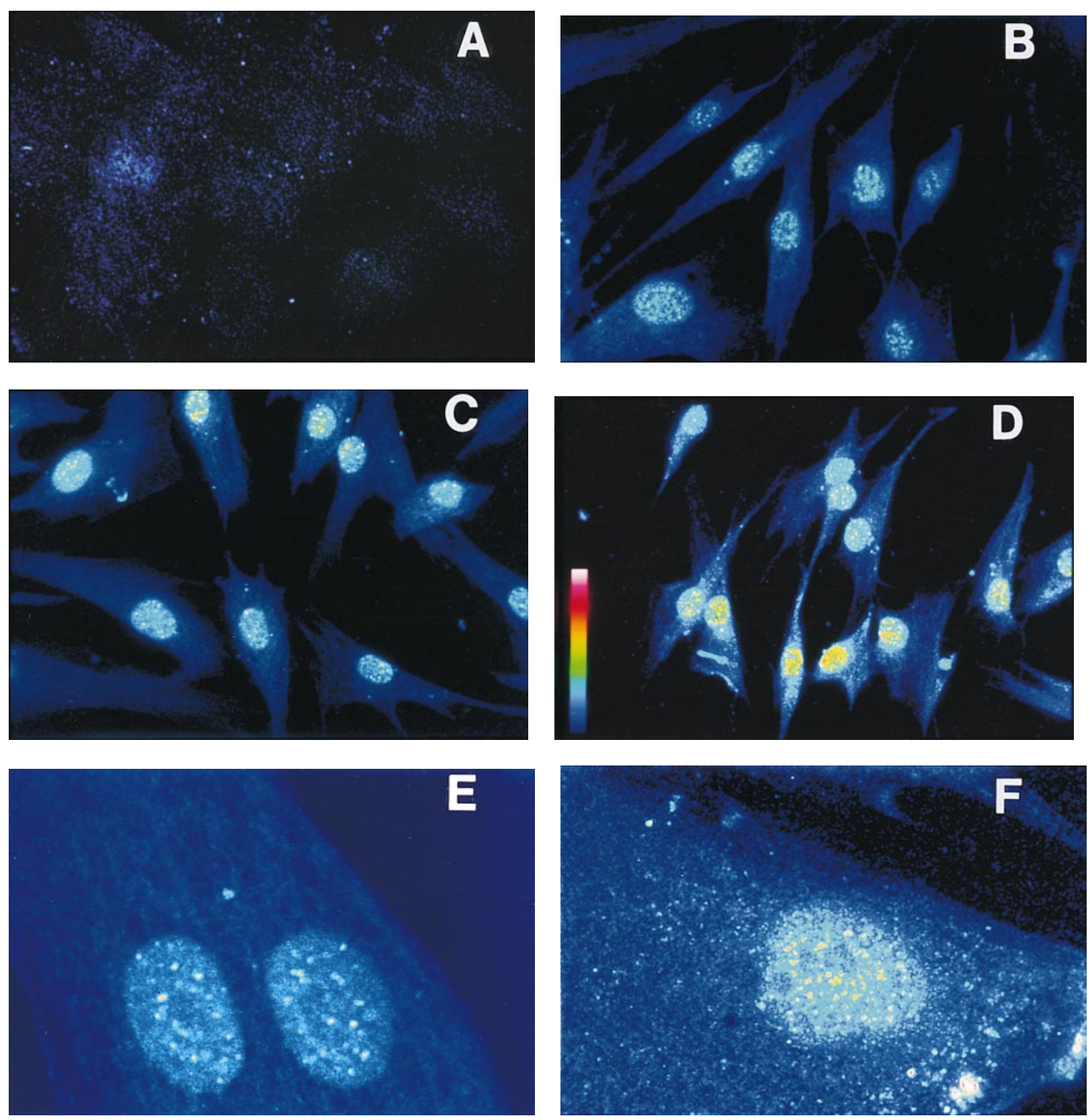

Figure 1. Appearance of nuclear HNE-protein adducts in hHSC. hHSC cultured on glass coverslips in Iscove's medium were deprived of serum and insulin for $24 \mathrm{~h}$. Cells were then left untreated $(A)$ or incubated with $10^{-6} \mathrm{M}(F)$ or $10^{-5} \mathrm{M} \mathrm{HNE}(B-E)$. At the indicated time points $(B, 5$ $\min ; C, 15 \mathrm{~min} ; A$ and $D-F, 30 \mathrm{~min}$ ), cells were fixed and permeabilized, then stained for indirect immunofluorescence with the monoclonal antibody $1 \mathrm{~g} 4 \mathrm{~h} 7$. Cells were then viewed with a laser scanner confocal microscope and six focal frames were taken along the $z$ axis at $1-\mu \mathrm{m}$ intervals and then merged to obtain a reconstituted image. $E$ was obtained by collecting a simple median focal frame of $1 \mu \mathrm{m}$. Fluorescence was transformed in pseudocolors (see reference scale proportional to the intensity of fluorescence in $D$ ). 
A

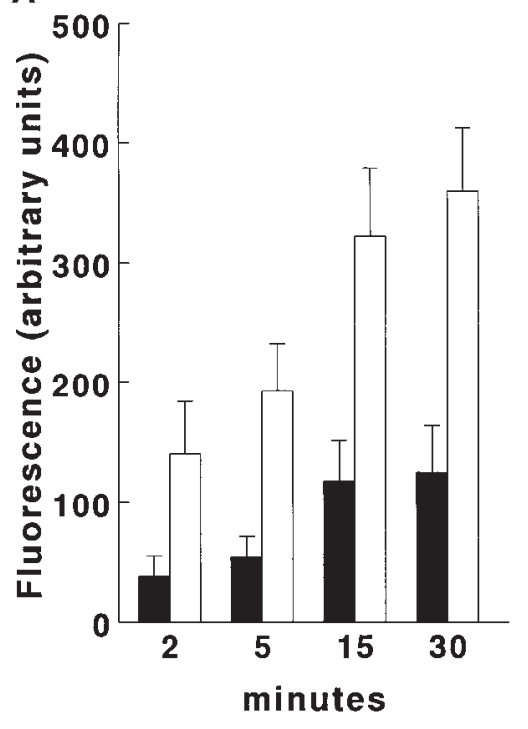

Figure 2. Nuclear localization of HNE-protein adducts is timedependent but independent on $\mathrm{Ca}^{2+}$ and defined signaling pathways. $(A)$ Intracellular localization of adduct fluorescence was evaluated by image analysis at the indicated time points in nuclear (open bars) and cytosolic (filled bars) compartments. Fluorescence values, corrected for their respective background values, were expressed as arbitrary units and as means \pm SD of $20-30$ determinations at any time point. (B) To study if nuclear HNEprotein adducts were related to $\mathrm{Ca}^{2+}$ homeostasis or specific signaling pathway, hHSC were deprived of serum and insulin for $24 \mathrm{~h}$ and, before addition of $10^{-5} \mathrm{M} \mathrm{HNE}$ for $30 \mathrm{~min}$, were left untreated or processed as

follows. To monitor dependence on $\mathrm{Ca}^{2+}$ homeostasis, serum-free and insulin-free medium (SFIF medium) was substituted with fresh $\mathrm{Ca}^{2+}$ deprived, $1 \mathrm{mM}$ EGTA supplemented SFIF medium just $5 \mathrm{~min}$ before HNE addition (EGTA). In some experiments, this procedure was preceded by 15 min of preincubation with $20 \mu \mathrm{M}$ EGTA-AM. Dependence on defined signaling pathways was studied by adding, before HNE, the following inhibitors: tyrphostin 23 (TYR 23) and herbimycin A (Herb.; inhibitors of receptor and nonreceptor tyrosine kinases, respectively), okadaic acid (Ok. Ac.; inhibitor of protein phosphatases), apigenin (Apig.; inhibitor of classic PKC isoforms), wortmannin (WT; inhibitor of PI3 kinase), PMA (depletion of classic PKC isoforms), or cycloheximide (C-Hex; inhibitor of de novo protein synthesis).

ing differences, filters were cohybridized with a ${ }^{32} \mathrm{P}$-labeled probe encoding for the ribosomal protein 36B4 (42).

Measurement of DNA synthesis. Confluent hHSC in 24-well dishes were washed with PBS and incubated in SFIF medium for $48 \mathrm{~h}$. The cells were then incubated with different concentrations of HNE for $24 \mathrm{~h}$. DNA synthesis was measured as the incorporation of $\left[{ }^{3} \mathrm{H}\right]$ thymidine as described elsewhere (43).

\section{Results}

We first investigated the formation of HNE-protein adducts in HSC by indirect immunofluorescence using monoclonal antibodies against HNE-histidine (33). Confocal laser microscopy analysis demonstrated an early nuclear localization of HNE adducts (Fig. 1). These adducts were evident, as defined spots of fluorescence, as early as 2-5 min after the addition of either $10^{-6}$ or $10^{-5} \mathrm{M}$ HNE. Computerized image analysis (Fig. $2 \mathrm{~A}$ ) indicated that nuclear fluorescence increased in a time-dependent fashion, reaching a plateau level after $30 \mathrm{~min}$ of incubation. Comparison of nuclear versus cytosolic fluorescence (Fig. $2 A$ ) indicated a 3:4 ratio in favor of the nuclear compartment. HNE-protein adduct generation and their nuclear localization occurred independently of intra- and extracellular $\mathrm{Ca}^{2+}$ movements and did not involve receptor or nonreceptor tyrosine kinases, protein phosphatases, or PI-3 kinase (Fig. 2 B). Depletion of classic isoforms of protein kinase $\mathrm{C}(\mathrm{PKC})$ or inhibition
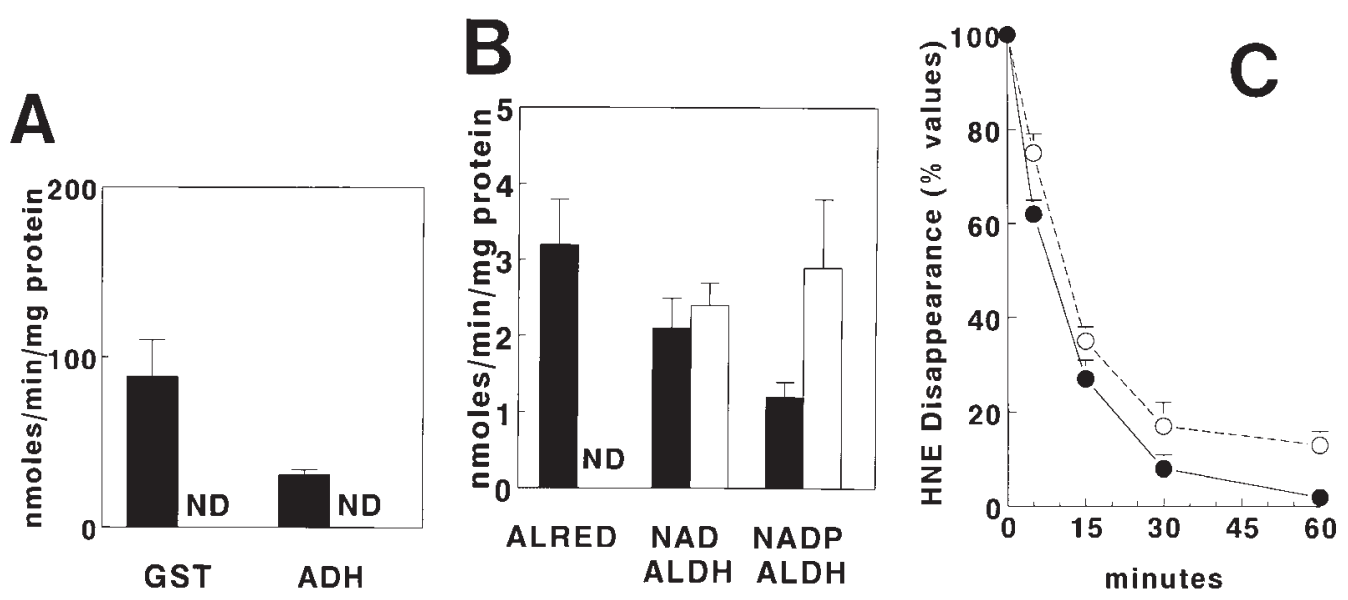

Figure 3. HNE metabolism in hHSC. ( $A$ and $B$ ) hHSC (open bars) and, for comparison, hepatocytes (filled bars) isolated from rat liver, were processed in order to obtain cytosolic fractions and to analyze HNE-metabolizing activities using HNE as substrate. Glutathione transferase activity $(G S T)$ was expressed as nmol of HNE consumed/min/ mg protein; inverse alcohol dehydrogenase $(A D H)$ and aldehyde reductase $(A L R E D)$ as nmol NAD $(\mathrm{P}) \mathrm{H}$ consumed/ $\mathrm{min} / \mathrm{mg}$ protein; and ALDH as nmol of $\mathrm{NADP}(\mathrm{P})$ reduced/ $\mathrm{min} / \mathrm{mg}$ protein. Values represent mean \pm SD of five experiments. $N D$, not detectable. $(C)$ Time-dependent HNE disappearance in cultured hHSC exposed to $10^{-5} \mathrm{M}$ HNE was measured, by means of an HPLC procedure, in the presence (open circles) or in the absence (filled circles) of cyanamide, an ALDH inhibitor. 


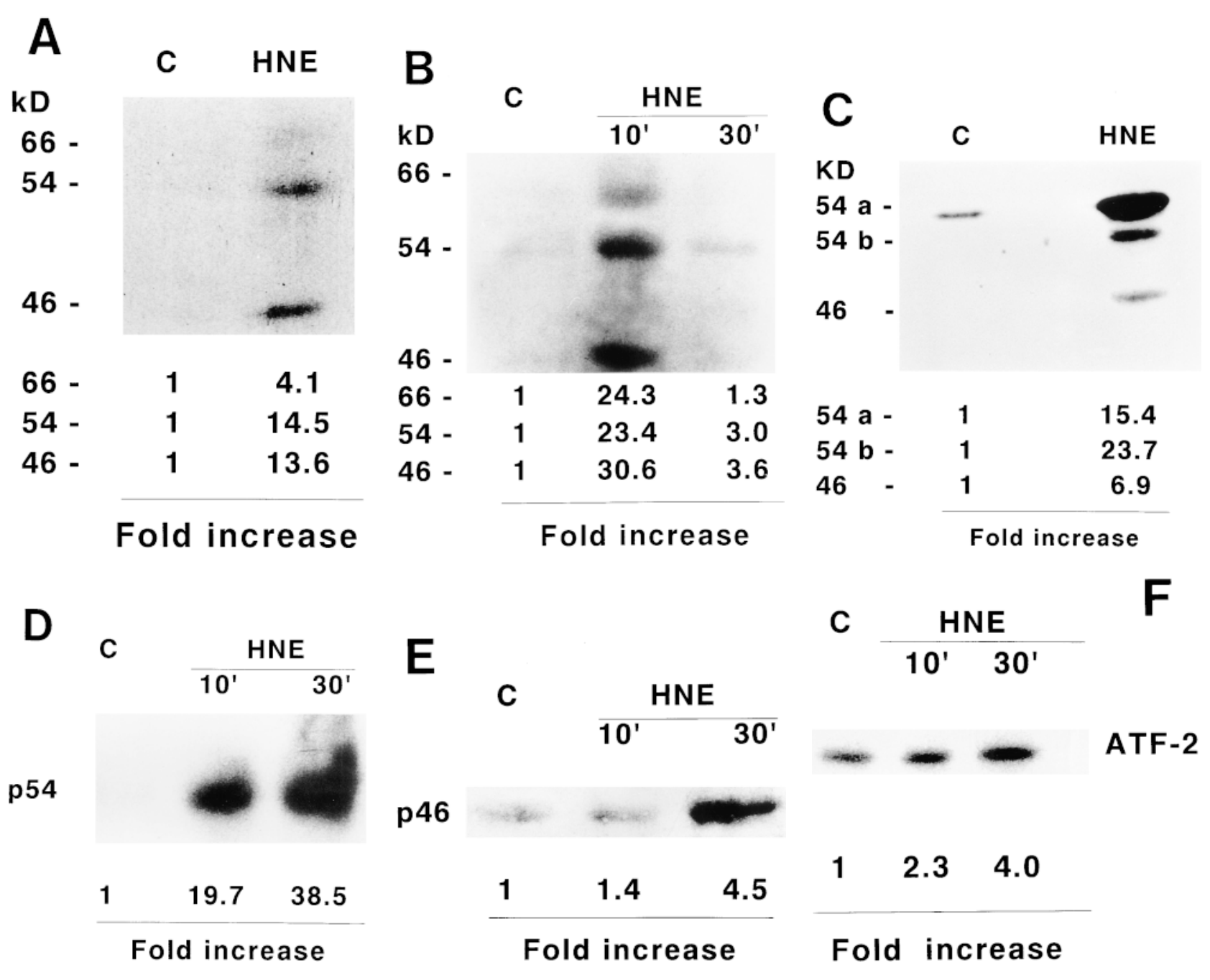

G $\begin{array}{r}\text { JNK activity } \\ \text { (fold increase) }\end{array}$
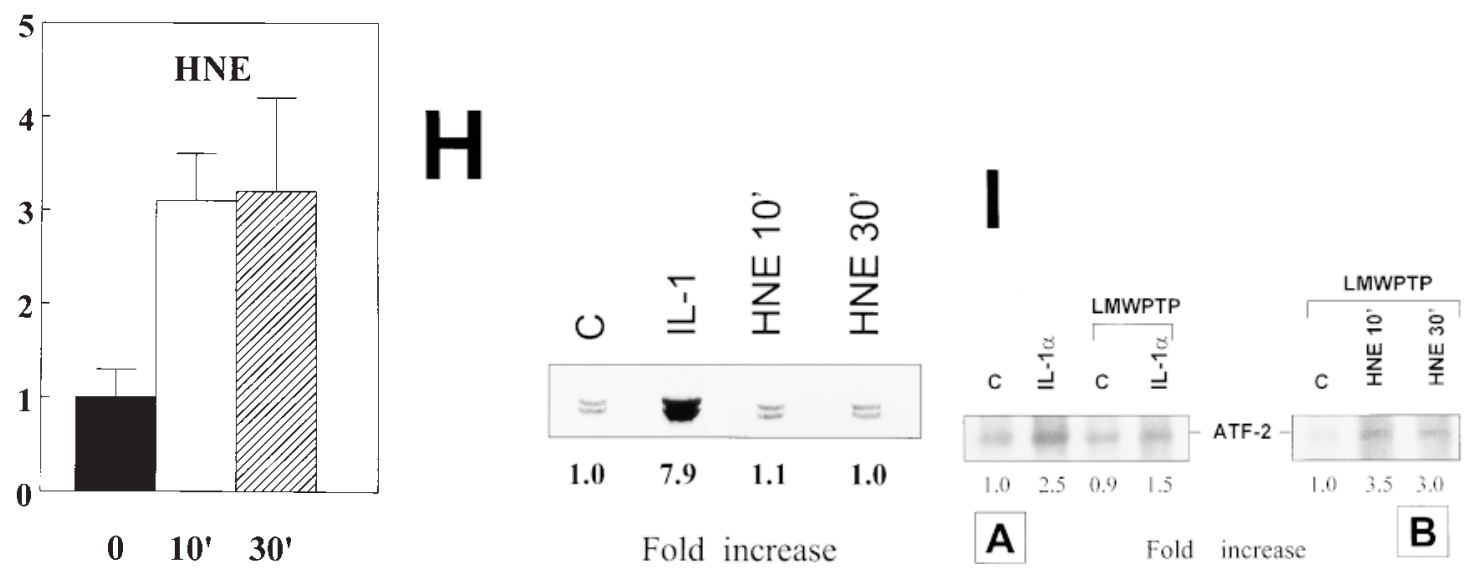

Figure 4. Identification of JNKs as targets for the action of HNE. hHSC were cultured in SFIF medium for $24 \mathrm{~h}$, left untreated $(C)$ or exposed to HNE $10^{-5} \mathrm{M}$ for different times and lysed to obtain NEs and cytosolic fractions. ( $A$ ) hHSC, exposed to HNE for 30 min, were analyzed for nuclear HNE-protein adducts by immunoblotting NEs with the monoclonal antibody $1 \mathrm{~g} 4 \mathrm{~h} 7$ against HNE-protein adducts. $(B)$ Cytosolic fractions from control cells $(C)$ and cells treated with HNE for 10 or $30 \mathrm{~min}$ were immunoblotted with the $1 \mathrm{~g} 4 \mathrm{~h} 7 \mathrm{antibody}$. (C) NEs were tested by immunoblotting with a polyclonal antibody recognizing JNK1 (p46 and p54 or JNK $\gamma$ ), JNK2 (p54 $\alpha$ ) and p54 $\beta$ (Santa Cruz Biotechnology) in control cells $(C)$ or hHSC treated with HNE for $30 \mathrm{~min}$. ( $D$ and $E$ ) NEs were immunoprecipitated using the same anti-JNKs antibody, $100 \mu \mathrm{g}$ of nuclear proteins were loaded for each lane, and isoforms at $54(D)$ and $46 \mathrm{kD}(E)$ were revealed using the $1 \mathrm{~g} 4 \mathrm{~h} 7$ antibody. $(F) \mathrm{JNK}$ activity was evaluated by using an immune complex kinase assay with ATF-2 as substrate. In this figure a representative experiment is shown, whereas in $G$, data 
of protein synthesis was also equally ineffective on the appearance of HNE-protein adducts in HSC nuclei (Fig. $2 \mathrm{~B}$ ).

Since the formation of HNE-protein adducts depends on HNE metabolism in a given cell type $(16,31,42)$ we evaluated this aspect in HSC (Fig. 3). hHSC were shown to be devoid of HNE-metabolizing glutathione- $S$-transferase and reverse alcohol dehydrogenase (Fig. $3 A$ ) which have been reported to be responsible for $90-95 \%$ of HNE enzymatic removal in liver parenchymal cells $(31,44)$. Aldehyde reductase was equally undetectable in these cells. Only a modest HNE metabolism by aldehyde dehydrogenase (ALDH) was detectable in hHSC (Fig. 3 B). However, $80-90 \%$ of HNE disappeared from the culture medium within 30 min (Fig. $3 \mathrm{C}$ ) even in the presence of the ALDH inhibitor cyanamide, thus indicating that in these cells formation of adducts largely exceeds HNE metabolism. Interestingly, kinetics of disappearance of HNE was in agreement with the appearance of fluorescent nuclear HNEprotein adducts.

To identify which proteins formed adducts with HNE, NEs were immunoblotted using the monoclonal antibody against HNE-histidine adducts. Two major nuclear adducts with molecular masses of 46 and $54 \mathrm{kD}$ and a minor adduct of $66 \mathrm{kD}$ were detected in hHSC treated with HNE (Fig. $4 A$ ). Immunoblotting of the corresponding cytosolic fractions revealed the presence of the same bands, the intensity of which decreased in a time-dependent fashion, suggesting nuclear translocation of the HNE-protein adducts (Fig. $4 \mathrm{~B}$ ). This observation and the apparent molecular weights of the proteins reacting with HNE (46 and $54 \mathrm{kD}$ ), compatible with those of JNKs (45), prompted us to investigate the possible involvement of this class of protein kinases. Nuclear proteins were immunoblotted using an anti-JNK antibody recognizing both the 54 and 46 isoforms (Fig. $4 \mathrm{C}$ ). A marked increase in the intensity of both bands was observed upon HNE stimulation, in agreement with a possible nuclear translocation of JNKs. To confirm the involvement of JNKs, NEs from control and HNE-treated cells were sequentially immunoprecipitated with anti-JNKs antibodies and blotted with antiadduct antibodies (Fig. 4, $D$ and $E)$. Exposure to HNE resulted in the appearance of HNE-JNK adducts in HSC nuclei, as evidenced by a 20-40-fold increase in the intensity of the band at $54 \mathrm{kD}$ (Fig. $4 \mathrm{D}$ ). In addition, prolonged exposure of the blots revealed that also the intensity of the band at $46 \mathrm{kD}$ was increased, although to a lesser extent (Fig. $4 E$ ). To establish whether JNK translocation to the nuclei is associated with increased enzymatic activity, NEs were used to measure JNK activity using recombinant ATF-2 as a substrate. These experiments (Fig. $4 F$ ) provided evidence for an HNE-induced increase in JNK enzymatic activity in HSC NEs. To establish whether the increase in JNK activity was brought about by phosphorylation of critical threonine and tyrosine residues, we performed Western blot analysis of NEs from HNE-treated cells using antibodies specifically recognizing phosphorylated JNK. Whereas IL- $1 \alpha$, used as a positive control, was able to induce an evident increase in phosphory- lated JNK isoforms, HNE had no effect (Fig. $4 G$ ). To further confirm that phosphorylation of JNK is not involved in the observed effects of HNE, samples were treated with recombinant LMW-PTP after immunoprecipitation with JNK antibodies. In fact, to achieve complete activation, phosphorylation of the TPY motif of JNK on threonine and tyrosine residue is required. LMW-PTP treatment greatly reduced the stimulatory effect of IL-1 on JNK activity, compatible with dephosphorylation of the critical tyrosine residue (Fig. $4 I$, panel $A$ ). In contrast, phosphatase treatment did not inhibit the increase in JNK activity in NEs of HSC exposed to HNE (Fig. 4I, panel B).

Proteins of the Jun family together with c-Fos and ATF-2 participate in the formation of AP-1, a transcriptional activator which regulates several genes (45). Activation of JNKs by HNE was associated with a biphasic increase in AP-1 DNA binding activity (Fig. $5 \mathrm{~A}$ ). A first increase was observed 15 min after HNE, whereas a second wave of increase in AP-1 binding activity was detected starting at 60-90 min after HNE. c-Jun was primarily involved in the early AP-1 complex (Fig. 5 $B)$, since the band at $15 \mathrm{~min}$ almost completely disappeared when NEs were preincubated with anti-c-Jun antibodies. However, the late AP-1 complexes were only partially inhibited by anti-c-Jun antibodies and almost unaffected by antic-Fos or anti-ATF-2 antibodies. To establish whether this delayed activation of AP-1 could be related to changes in $c$-jun expression, we analyzed $c$-jun mRNA levels in response to HNE. As shown in Fig. $5 C$, a marked upregulation of $c$-jun mRNA levels was observed 30-60 min after exposure to the aldehyde. This behavior is in agreement with the observed increase in JNK activity.

The involvement of ERK, potentially leading to AP-1 increase through c-Fos phosphorylation, was also evaluated. However, HNE did not modify ERK activity (Fig. 5 D), nor did it stimulate DNA synthesis (Fig. 5 E). Along these lines, HNE did not stimulate $c$-fos mRNA expression (data not shown). A known target of oxidative stress is represented by $\mathrm{NF}-\kappa \mathrm{B}$, which usually increases early after exposure to ROS. However, HNE did not affect NF-кB binding activity at any of the time points tested (Fig. $5 F$ ).

\section{Discussion}

In this study we report that HNE, an aldehydic end product of lipid peroxidation, is able to specifically interact with JNK isoforms, to lead to their nuclear translocation and activation, and to elicit a JNK/c-jun/AP-1 pathway. HNE and other 4-hydroxyalkenals have been detected unequivocally in fibrotic livers by several laboratories (17-25). These aldehydes may originate during chronic liver diseases either as a consequence of lipid peroxidation induced by prooxidant agents or after activation of inflammatory cells $(5-7,16,18)$. HNE has been shown to regulate gene expression in HSC, the key effectors in hepatic fibrogenesis. In particular, different laboratories have reported that HNE and other structurally related aldehydes upregulate

Fig. 4 legend (Continued)

are expressed as mean \pm SD of three different experiments. $(H)$ The state of phosphorylation of JNK isoforms was evaluated in NEs from control and HNE-treated cells as well as from IL-1 $\alpha$-treated hHSC as positive control by using specific antibodies against phosphorylated JNK isoforms. (I) Cell lysates from HSC treated with IL-1 $\alpha(A)$ or NEs from cells treated with HNE $(B)$ were immunoprecipitated with anti-JNK antibodies and incubated with or without LMW-PTP. At the end of the incubation, the immunobeads were extensively washed and JNK assay was performed as described in Methods. Fold increase was determined by means of laser densitometry. 

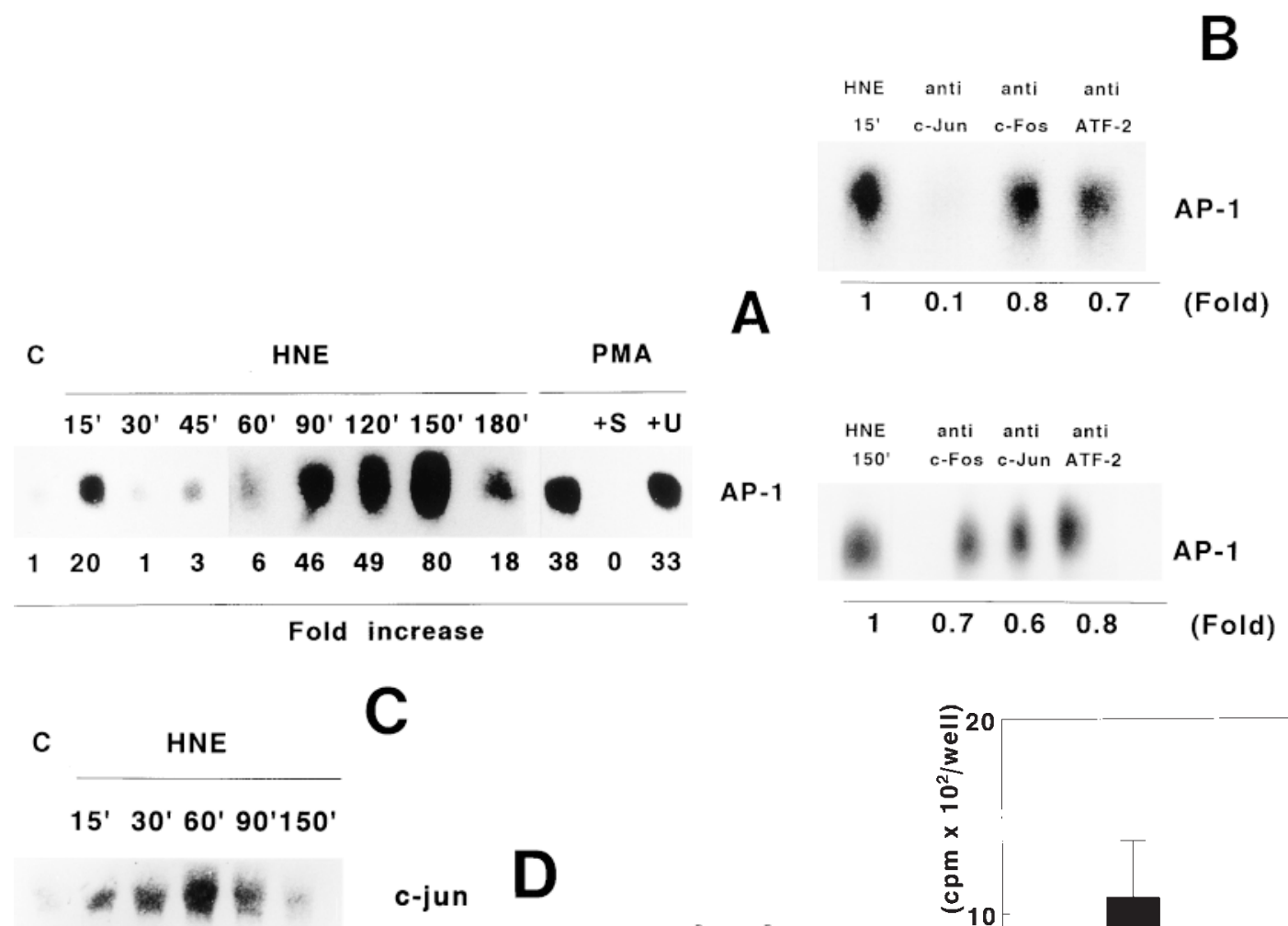

$\begin{array}{llllll}1 & 4.1 & 6.6 & 12 & 6.7 & 1.9\end{array}$

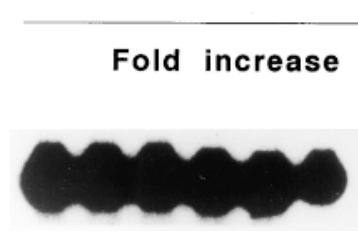

36B4

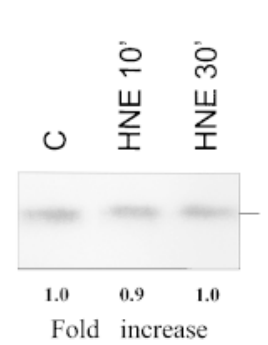

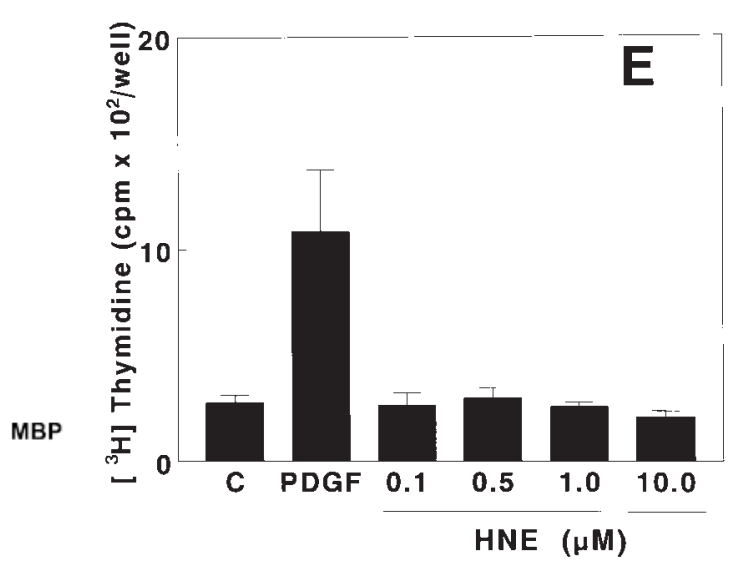

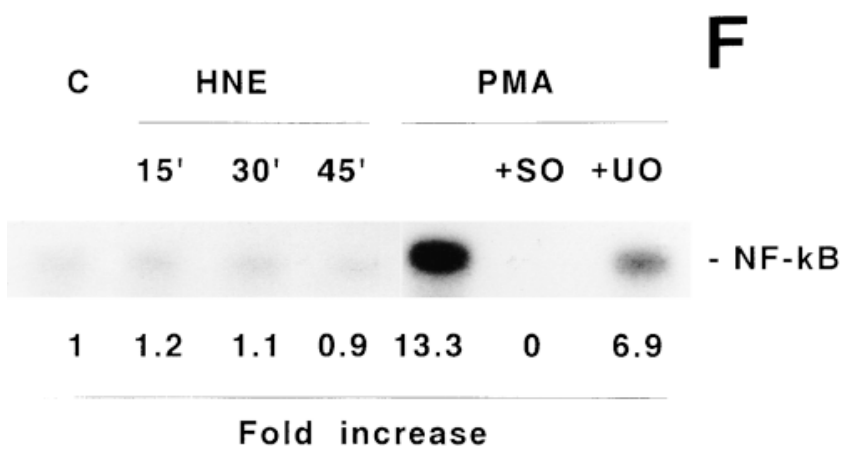

Figure 5. Effect of HNE on AP-1 and NF-кB transcription factors, c-Jun protooncogene, ERK activity, and DNA synthesis. hHSC were cultured for $24 \mathrm{~h}$ in SFIF medium, left untreated $(C)$, exposed to $10^{-6} \mathrm{M}$ HNE (unless otherwise stated) or to other agonists (see below) for different times and then processed as follows. $(A)$ NEs were assayed for AP-1 binding activity in control and HNE-treated hHSC using EMSA. For control purposes, HSC were incubated with $50 \mathrm{ng} / \mathrm{ml}$ of PMA for 45 min and competition experiments were performed by incubating NEs with the labeled probe in the presence of 100 -fold excess of unlabeled AP-1 oligonucleotide (specific oligonucleotide or $S$ ) or unlabeled NF-кB oligonucleotide (unspecific oligonucleotide or $U$ ) for 45 min. (B) NEs from HNE-treated cells at 15 (top) and $150 \mathrm{~min}$ (bottom) were either left untreated or pretreated with antibodies directed against c-Jun, c-Fos, and ATF-2, respectively, and then assayed for AP-1 binding activity using EMSA. $(C)$ Total RNA was isolated at the indicated time points and mRNA levels for c-Jun were evaluated by standard Northern blot analysis. A probe encoding for the microsomal protein 36B4 was used to monitor equal loading of samples. $(D)$ ERK activity was evaluated in nuclear extracts from control and HNE-treated cells by using MBP as substrate. $(E)$ DNA synthesis was evaluated by incorporation of $\left[{ }^{3} \mathrm{H}\right]$ thymidine at the end of $24 \mathrm{~h}$ of incubation in the absence of stimuli $(C)$ or in the presence of PDGF-BB $10 \mathrm{ng} / \mathrm{ml}$ or different HNE concentrations. $(F)$ EMSA for NF-kB on NEs from control $(C)$ and HNE-treated cells. For control purposes, HSC were incubated with $50 \mathrm{ng} / \mathrm{ml}$ of PMA for 45 min and competition experiments were performed by incubating NEs with the labeled probe in the presence of 100 -fold excess of unlabeled NF-kB oligonucleotide (specific oligonucleotide or $S O$ ) or unlabeled AP-1 oligonucleotide (unspecific oligonucleotide or $U O$ ). Fold increase was determined by means of laser densitometry.

the expression and secretion of procollagen I, thus directly contributing to the deposition of extracellular matrix during fibrogenesis $(6,26,27)$. Data from this study elucidate for the first time the signaling pathways elicited by HNE and establish a potential link with the regulation of gene expression in HSC. Moreover, our studies indicate HSC as a preferential target for
HNE-mediated effects in the liver in view of their inability to rapidly metabolize this aldehyde.

HNE is known to act as a nonoxidant and highly reactive compound able to form adducts with cysteine, lysine and, in particular, histidine by a Michael type reaction $(16,33)$. The data herein reported indicate that JNK nuclear translocation 
and activation are strictly related to the formation of these adducts. In turn, JNK effectively activated downstream events such as AP-1 binding activity and increased expression of $c$-jun protooncogene. This mechanism of action differs from the one elicited by ROS on so-called redox sensors, which has been mostly related to the oxidation of critical sulfhydryl groups and to the alteration of intracellular redox state (8-11). This oxidizing mechanism accounts for the recently reported activation of JNKs by hydrogen peroxide and nitric oxide (36). Importantly, HNE concentrations used in the present study are not toxic for hHSC and have been shown to be reached in vivo or in vitro in whole livers or isolated hepatocytes undergoing lipid peroxidation (for review see reference 16).

Activation of JNK has been reported to occur after exposure to ultraviolet light, heat shock, and cytokines (45-47). All of these stimuli operate through small $G$ proteins of the Ras superfamily and sequential activation of the protein kinases MEKK1 and MEKK4 (45-47). This cascade is likely to be operating also when JNK activation is induced by hydrogen peroxide and nitric oxide (36). In this study we provide evidence that translocation and activation of JNK in HNE-treated cells occur independently of phosphorylation. Translocation of JNK with presumably low enzymatic activity, due to the lack of phosphorylation, may also explain the quantitative discrepancy between the amount of JNK translocated to the nucleus and the increase in JNK activity. Interestingly, the presence of multiple histidine residues particularly in the p54 ${ }^{\mathrm{INK}}$ isoform (48) may provide the basis for a conformational change potentially responsible for nuclear translocation, and possibly activation, in the absence of phosphorylation. Data reported in Fig. 4, $H$ and $I$, further support the concept of a direct interaction between HNE and JNK isoforms since JNKs were not phosphorylated and the pretreatment of hHSC extracts with a LMW-PTP did not affect JNK activation, then excluding an involvement of upstream kinases. Moreover, the observation that HNE does not affect the activation of the transcription factor NF-кB supports the concept that HNE does not induce JNK phosphorylation. Indeed, it has been shown recently that the activation of MEKK1 leads also to the activation of the I $\mathrm{B} \alpha$ kinase complex and then to an increased NF- $\mathrm{B}$ binding activity (49). In addition, the lack of effect of $\mathrm{HNE}$ on $\mathrm{NF}-\kappa \mathrm{B}$, a classic redox sensor, further suggests that this aldehyde acts by a nonoxidant mechanism.

HNE elicited in hHSC a biphasic increase in AP-1 binding activity. The first peak, which occurred immediately after stimulation, presumably involved phosphorylation of preexisting c-Jun/AP-1 proteins (45) as indicated by experiments performed using specific anti-c-Jun antibodies. Accordingly, early activation of JNKs induced by HNE (10 min) temporally preceded the first peak of AP-1. The second wave possibly involved increased synthesis of c-Jun, mediated by the interaction of AP-1 with the $c$-jun promoter, as indicated by Northern blot experiments showing increased c-jun expression 30-60 min after stimulation with HNE (45). However, c-Jun only partially contributed to the formation of the second peak of AP-1 activity, since specific antibodies inhibited formation of the complex only by $30-40 \%$. It remains to be established whether the second peak of AP-1 may be directly related to HNE-JNK interaction and activation.

Regulation of procollagen I expression, the major biologic effect of HNE on hHSC, has been the focus of extensive investigation, and several transcription factors and regulatory ele- ments have been shown to be implicated (for review see reference 50). In particular, AP-1 has been shown to be relevant for the induction of procollagen type I gene expression in HSC using a 360-bp human collagen type I promoter-enhancer construct and a reporter gene (51). Deletion of the AP-1 motif from this construct drastically reduced procollagen $\alpha 1(\mathrm{I})$ transcription. Furthermore, treatment of HSC extracts with antisera against c-Jun was much more effective in reducing AP-1 binding to the procollagen I promoter than antisera against c-Fos, suggesting a major role for c-Jun in the formation of the AP-1 complex (51). Therefore, the effects on JNK by HNE reported herein may be relevant for the understanding of the biologic effects so far ascribed to HNE.

The precise role of JNKs in liver physiology and pathophysiology, as recently reviewed (52), is presently unclear, although recent studies suggest that this pathway could be involved in several biologic processes. JNK activation after carbon tetrachloride-induced oxidative stress has been linked to inhibition of hepatocyte proliferation, and induction of apoptosis (53), and activation of this pathway has also been described after warm reperfusion after experimental liver transplantation (54). On the other hand, JNK activation has been reported to occur after partial hepatectomy and to be a relevant event in hepatic regeneration (55). Moreover, c-Jun null mice show several developmental abnormalities, including hepatic agenesia, suggesting that c-Jun is required for liver development (56). Although limited to a nonparenchymal liver cell type, the data herein reported indicate a possible additional role for JNK in the development of liver fibrogenesis, and provide a mechanism of action for the biological effect of HNE on hHSC.

In conclusion, this study identifies for the first time a novel nonoxidant mechanism linking oxidative stress to nuclear signaling in hHSC through the action of an aldehydic end product of lipid peroxidation. This mechanism may be relevant in sustaining oxidative stress-mediated effects in conditions of chronic liver diseases.

\section{Acknowledgments}

This paper is dedicated to the memory of Prof. Hermann Esterbauer, an outstanding scientist in the field of biological effects of aldehydic end products of lipid peroxidation, a nice teacher, and for many years a good friend.

The authors are grateful to Dr. Goutam Ghosh Choudhury (Department of Medicine, University of Texas Health Science Center, San Antonio, TX) for his helpful suggestions.

This study was supported by Ministero della Università e della Ricerca Scientifica e Tecnologica (National project on Liver Cirrhosis and Viral Hepatitis), by the Associazione Italiana Ricerca sul Cancro (AIRC), by the Consiglio Nazionale delle Ricerche (ACRO Project and Progetto Finalizzato Biotecnologie), by the Italian Liver Foundation (Florence), by the Austrian Science Foundation (Project SFB 709), and by the Association for International Cancer Research (Project 96-10).

\section{References}

1. Blomhoff, R., and K. Wake. 1991. Perisinusoidal stellate cells of the liver: important roles in retinol metabolism and fibrosis. FASEB (Fed. Am. Soc. Exp. Biol.) J. 5:271-277.

2. Friedman, S.L. 1993. The cellular basis of hepatic fibrosis. N. Engl. J. Med. 328:1828-1835.

3. Maher, J.J., and R.F. McGuire. 1990. Extracellular matrix gene expression increases preferentially in rat lipocytes and sinusoidal endothelial cells dur- 
ing hepatic fibrosis in vivo. J. Clin. Invest. 86:1641-1649.

4. Bissel, D.M. 1992. Lipocyte activation and hepatic fibrosis. Gastroenterology. 102:1803-1805.

5. Britton, R.S., and B.R. Bacon. 1994. Role of free radicals in liver diseases and hepatic fibrosis. Hepatogastroenterology. 41:343-348.

6. Tsukamoto, H., R. Rippe, O. Niemela, and M. Lin. 1995. Role of oxidative stress in activation of Kupffer and Ito cell. J. Gastroenterol. Hepatol. 10: S50-S53.

7. Pietrangelo, A. 1996. Metals, oxidative damage and fibrogenesis. Semin. Liver Dis. 16:13-30.

8. Crawford, D., I. Zbinden, P. Amstad, and P. Cerruti. 1988. Oxidant stress induces the protooncogenes c-fos and c-myc in mouse epidermal cells. Oncogene. 3:27-32.

9. Shibanuma, M., T. Kuroki, and K. Nose. 1988. Induction of DNA replication and expression of protooncogenes c-myc and c-fos in quiescent Balb/3T3 cells by xanthine/xanthine oxidase. Oncogene. 3:17-21.

10. Abate, C., L. Patel, F.J. Rauscher, III, and T. Curran. 1990. Redox regulation of Fos and Jun DNA binding activity in vitro. Science. 249:1157-1161.

11. Schreck, R., P. Rieber, and P.A. Bauerle. 1991. Reactive oxygen intermediates as apparently widely used messengers in the activation of the NF-kB transcription factor and HIV-1. EMBO (Eur. Mol. Biol. Organ.) J. 10:2247-2258.

12. Halliwell, B., and J.M.C. Gutteridge. 1989. Free Radicals in Biology and Medicine. 2nd edition. Clarendon Press, Oxford.

13. Arnaiz, S.L., S. Llesuy, J.C. Cutrin, and A. Boveris. 1995. Oxidative stress by acute acetaminophen administration in mouse liver. Free Rad. Biol. Med. 19:303-310.

14. Slater, T.F. 1982. Free radical mechanisms in tissue injury. Biochem. J. 222:1-15.

15. Poli, G., E. Albano, and M.U. Dianzani. 1987. The role of lipid peroxidation in liver damage. Chem. Phys. Lipids. 45:117-142.

16. Esterbauer, H., R.J. Schaur, and H. Zollner. 1991. Chemistry and biochemistry of 4-hydroxynonenal, malondialdehyde and related aldehydes. Free Rad. Biol. Med. 11:81-128.

17. Bedossa, P., K. Houglum, C. Trauwein, A. Holstege, and M. Chojkier. 1994. Stimulation of collagen $\alpha 1$ (I) gene expression is associated with lipid peroxidation in hepatocellular injury. A link to tissue fibrosis? Hepatology. 19: 1262-1271.

18. Poli, G., and M. Parola. 1997. Oxidative damage and fibrogenesis. Free Rad. Biol. Med. 22:287-305.

19. Parola, M., G. Leonarduzzi, F. Biasi, E. Albano, M.E. Biocca, G. Poli, and M.U. Dianzani. 1992. Vitamin E dietary supplementation protects against carbon tetrachloride-induced chronic liver damage and cirrhosis. Hepatology. 16:1014-1021.

20. Kamimura, S., K. Gaal, R.S. Britton, B.R. Bacon, G. Triadafilopoulos, and H. Tsukamoto. 1992. Increased 4-hydroxynonenal levels in experimental alcoholic liver disease: association of lipid peroxidation with liver fibrosis. Hepatology. 16:448-453.

21. Niemela, O., S. Parkkila, S. Ylä-Herttuala, J. Villanueva, B. Ruebner, and C. Halsted. 1994. Sequential acetaldehyde production, lipid peroxidation and fibrogenesis in micropig model of alcohol induced liver injury. Hepatology. 22:1208-1214.

22. Tsukamoto, H., W. Horne, S. Kamimura, O. Niemelä, S. Parkkila, S. Ylä-Herttuala, and G.M. Brittenham. 1995. Experimental liver cirrhosis induced by alcohol and iron. J. Clin. Invest. 96:620-630.

23. Parola, M., G. Leonarduzzi, G. Robino, E. Albano, G. Poli, and M.U. Dianzani. 1996. On the role of lipid peroxidation in the pathogenesis of liver damage induced by long-standing cholestasis. Free Rad. Biol. Med. 20:351-359.

24. Paradis, V., M. Kollinger, M. Fabre, A. Holstege, T. Poynard, and P. Bedossa. 1997. In situ detection of lipid peroxidation by-products in chronic liver diseases. Hepatology. 26:135-142.

25. Venkataramani, A., K. Houglum, K. Lyche, and M. Chojkier. 1996. Oxidative stress mediates activation of stellate cells and fibrogenesis in patients with chronic hepatitis C: beneficial effects of D- $\alpha$-tocopherol. Hepatology. 24: 93a. (Abstr.)

26. Parola, M., M. Pinzani, A. Casini, E. Albano, G. Poli, A. Gentilini, P. Gentilini, and M.U. Dianzani. 1993. Stimulation of lipid peroxidation or 4-hydroxynonenal treatment increases procollagen $\alpha$ (I) gene expression and synthesis in human liver fat-storing cells. Biochem. Biophys. Res. Commun. 194:1044-1050.

27. Parola, M., M. Pinzani, A. Casini, G. Leonarduzzi, F. Marra, A. Caligiuri, E. Ceni, P. Biondi, G. Poli, and M.U. Dianzani. 1996. Induction of procollagen type I gene expression and synthesis in human hepatic stellate cells by 4-hydroxy-2,3-nonenal and other 4-hydroxy-2,3-alkenals is related to their molecular structure. Biochem. Biophys. Res. Commun. 222:261-264.

28. Maher, J.J., C. Tzagarakis, and A. Gimenez. 1994. Malondialdehyde stimulates collagen production by hepatic lipocytes only upon activation in primary culture. Alcohol Alcohol. 29:605-610.

29. Casini, A., M. Pinzani, S. Milani, C. Grappone, G. Galli, A.M. Jezequel, D. Schuppan, C.M. Rotella, and C. Surrenti. 1993. Regulation of extracellular matrix synthesis by transforming growth factor $\beta 1$ in human fat-storing cells. Gastroenterology. 10:245-253.

30. Marra, F., A.J. Valente, M. Pinzani, and H.E. Abboud. 1993. Cultured human liver fat-storing cells produce monocyte chemotactic protein 1 . Regulation by proinflammatory cytokines. J. Clin. Invest. 92:1674-1680.
31. Leonarduzzi, G., M. Parola, G. Muzio, A. Garramone, M. Maggiora, G. Robino, G. Poli, M.U. Dianzani, and R.A. Canuto. 1995. Hepatocellular metabolism of 4-hydroxy-2,3-nonenal is impaired in conditions of chronic cholestasis. Biochem. Biophys. Res. Commun. 214:669-675.

32. Poli, G., G. Cecchini, F. Biasi, E. Chiarpotto, R.A. Canuto, M.E. Biocca, G. Muzio, H. Esterbauer, and M.U. Dianzani. 1986. Resistance to oxidative stress by hyperplastic and neoplastic rat liver tissue monitored in terms of unpolar and medium polar carbonyls. Biochim. Biophys. Acta. 883:207-214.

33. Waeg, G., G. Dimsity, and H. Esterbauer. 1996. Monoclonal antibodies for detection of 4-hydroxynonenal modified proteins. Free Rad. Res. 25:149-159.

34. Andrew, N.C., and D.V. Faller. 1991. A rapid micropreparation technique for extraction of DNA-binding proteins from limiting numbers of mammalian cells. Nucl. Acids Res. 19:2499.

35. Laemmli, U.K. 1970. Cleavage of structural proteins during the assembly of the head of bacteriophage $\mathrm{T}_{4}$. Nature. 227:680-685.

36. Lo, Y.Y.C., J.M.S. Wong, and T.F. Cruz. 1996. Reactive oxygen species mediate cytokine activation of c-Jun $\mathrm{NH}_{2}$-terminal kinases. J. Biol. Chem. 271: 15703-15707.

37. Su, X.-D., N. Taddei, M. Stefani, G. Ramponi, and P. Nordlund. 1994. The crystal structure of a low-molecular-weight phosphotyrosine protein phosphatase. Nature. 370:575-578.

38. Stefani, M., A. Caselli, M. Bucciardini, L. Pazzagli, F. Dolfi, G. Camici, G. Manao, and G. Ramponi. 1993. Dephosphorylation of tyrosine phosphorylated synthetic peptides by rat liver phosphotyrosine protein phosphatase isoenzymes. FEBS Lett. 326:131-134.

39. Chirgwin, J.M., A.E. Pryzbyla, R.J. McDonald, and W.J. Rutter. 1979. Isolation of biologically active ribonucleic acid from sources enriched in ribonuclease. Biochemistry. 18:5294-5299.

40. Sambrook, J., E.F. Fritsch, and T. Maniatis. 1989. Analysis of RNA. In Molecular Cloning: A Laboratory Manual. J. Sambrook, E.F. Fritsch, and T. Maniatis, editors. Cold Spring Harbor Laboratory, Cold Spring Harbor, NY. 7.37-7.52

41. Mallat, A., A.-M. Préaux, C. Serradeil-Le Gal, D. Raufaste, C. Gallois, D. A. Brenner, C. Bradham, J. Maclouf, V. Iourgenko, L. Fouassier, et al. 1996. Growth inhibitory properties of endothelin-1 in activated human hepatic stellate cells: a cyclic adenosine monophosphate-mediated pathway. Inhibition of both extracellular signal-regulated kinase and c-Jun kinase and upregulation of endothelin B receptors. J. Clin. Invest. 98:2771-2778.

42. Bassett, P., J.P. Bellocq, C. Wolf, I. Stolle, P. Hutin, J.M. Limacher, O.L. Podhajcer, R.P. Chenard, M.C. Rio, and P. Chambon. 1990. A novel metalloproteinase gene specifically expressed in stromal cells of breast carcinoma. $\mathrm{Na}$ ture. 348:699-704.

43. Pinzani, M., L. Gesualdo, G.M. Sabbah, and H.E. Abboud. 1989. Effects of platelet-derived growth factor and other polypeptide mitogens on DNA synthesis and growth of cultured rat liver fat-storing cells. J. Clin. Invest. 84:1786-1793.

44. Canuto, R.A., M. Ferro, G. Muzio, A.M. Bassi, G. Leonarduzzi, M. Maggiora, D. Adamo, G. Poli, and M.U. Dianzani. 1994. Role of aldehyde metabolizing enzymes in mediating effects of aldehyde products of lipid peroxidation in liver cells. Carcinogenesis. 15:1359-1364.

45. Karin, M. 1995. The regulation of AP-1 activity by mitogen-activated protein kinases. J. Biol. Chem. 270:16483-16486.

46. Derijard, B., M. Hibi, I.-H. Wu, T. Barret, B. Su, T. Deng, M. Karin, and R.J. Davis. 1994. JNK1: a protein kinase stimulated by UV light and Ha-ras that binds and phosphorylates the c-Jun activation domain. Cell. 76:1025-1037.

47. Kyriakis, J.M., and J. Avruch. 1996. Sounding the alarm: protein kinase cascades activated by stress and inflammation. J. Biol. Chem. 271:24313-24316.

48. Kallunki, T., B. Su, I. Tsigelny, H.K. Sluss, B. Derijard, G. Moore, R. Davis, and M. Karin. 1994. JNK2 contains a specificity-determining region responsible for efficient c-Jun binding and phosphorylation. Genes Dev. 8:2996-3007.

49. Lee, F.K., J. Hagler, Z.J. Chen, and T. Maniatis. 1997. Activation of the $\mathrm{I} \kappa \mathrm{B} \alpha$ kinase complex by MEKK1, a kinase of the JNK pathway. Cell. 88:213-222.

50. Brenner, D.A., R.A. Rippe, K. Rhodes, J.F. Trotter, and M. Breindl. 1994. Fibrogenesis and type I collagen gene regulation. J. Lab. Clin. Med. 124: 755-760.

51. Armendariz-Borunda, J., C.P. Simkevich, N. Roy, R. Raghow, A.H Kang, and J.M. Seyer. 1994. Activation of Ito cells involves regulation of AP-1 binding proteins and induction of type I collagen gene expression. Biochem. J. 304:817-824.

52. Marra, F. 1997. Ischemia-reperfusion: the liver under stress. Hepatology. 25:1276-1278

53. Mendelson, K.G., L.-R. Contois, S.G. Tevosian, R.J. Davis, and K.E. Paulson. 1996. Independent regulation of JNK/p38 mitogen-activated protein kinases by metabolic oxidative stress in the liver. Proc. Natl. Acad. Sci. USA. 93 : 12908-12913.

54. Bradham, C.A., R.F. Stachlewitz, W. Gao, T. Qian, S. Jayadev, G. Jenkins, Y. Hannun, J.J. Lemasters, R.G. Thurman, and D.A. Brenner. 1997. Reperfusion after liver transplantation in rats differentially activates the mitogenactivated protein kinases. Hepatology. 25:1128-1135.

55. Westwick, J.K., C. Weitzel, H.L. Leffert, and D.A. Brenner. 1995. Activation of Jun kinase is an early event in hepatic regeneration. J. Clin. Invest. 95: 803-810.

56. Hilberg, F.A., A. Aguzzi, N. Howells, and E.F. Wagner. 1993. c-Jun is essential for normal mouse development and hepatogenesis. Nature. 365:179-181. 